\title{
The addition of a sagittal image fusion improves the prostate cancer detection in a sensor-based MRI /ultrasound fusion guided targeted biopsy
}

Karsten Günzel ${ }^{1 \dagger}$, Hannes Cash ${ }^{1 *}$, John Buckendahl ${ }^{1}$, Maximilian Königbauer ${ }^{1}$, Patrick Asbach², Matthias Haas², Jörg Neymeyer ${ }^{1}$, Stefan Hinz ${ }^{1}$, Kurt Miller ${ }^{1}$ and Carsten Kempkensteffen ${ }^{1}$

\begin{abstract}
Background: To explore the diagnostic benefit of an additional image fusion of the sagittal plane in addition to the standard axial image fusion, using a sensor-based MRI/US fusion platform.

Methods: During July 2013 and September 2015, 251 patients with at least one suspicious lesion on mpMRI (rated by PI-RADS) were included into the analysis. All patients underwent MRI/US targeted biopsy (TB) in combination with a 10 core systematic prostate biopsy (SB). All biopsies were performed on a sensor-based fusion system. Group A included 162 men who received TB by an axial MRI/US image fusion. Group B comprised 89 men in whom the TB was performed with an additional sagittal image fusion.

Results: The median age in group A was 67 years (IQR 61-72) and in group B 68 years (IQR 60-71). The median PSA level in group A was $8.10 \mathrm{ng} / \mathrm{ml}$ (IQR 6.05-14) and in group B $8.59 \mathrm{ng} / \mathrm{ml}$ (IQR 5.65-12.32). In group A the proportion of patients with a suspicious digital rectal examination (DRE) (14 vs. 29\%, $p=0.007)$ and the proportion of primary biopsies (33 vs 46\%, $p=0.046$ ) were significantly lower. The rate of PI-RADS 3 lesions were overrepresented in group A compared to group B (19 vs. 9\%; $p=0.044$ ). Classified according to PI-RADS 3, 4 and 5, the detection rates of TB were $42,48,75 \%$ in group A and 25, 74, 90\% in group B. The rate of PCa with a Gleason score $\geq 7$ missed by TB was 33\% (18 cases) in group $A$ and $9 \%$ ( 5 cases) in group B; $p$-value 0.072 . An explorative multivariate binary logistic regression analysis revealed that PI-RADS, a suspicious DRE and performing an additional sagittal image fusion were significant predictors for PCa detection in TB. 9 PCa were only detected by TB with sagittal fusion (sTB) and STB identified 10 additional clinically significant PCa (Gleason $\geq 7$ ).
\end{abstract}

Conclusion: Performing an additional sagittal image fusion besides the standard axial fusion appears to improve the accuracy of the sensor-based MRI/US fusion platform.

Keywords: Multiparametric magnetic resonance imaging, Targeted biopsy, Prostate cancer detection, MRI/US fusion biopsy

\footnotetext{
* Correspondence: hannes.cash@charite.de

Karsten Günzel and Hannes Cash are co-first authors.

'Equal contributors

'Department of Urology, Charité - University Medicine Berlin,

Hindenburgdamm 30, 12203 Berlin, Germany

Full list of author information is available at the end of the article
} 


\section{Background}

Prostate cancer $(\mathrm{PCa})$ is the most common malignancy of men and the only tumour, which is diagnosed according to the guidelines by untargeted systematic biopsies of the entire organ [1,2]. Because prostate cancer is often not visualized in conventional transrectal ultrasound, there is a risk to miss clinically significant $\mathrm{PCa}($ Gleason $\geq 7$ ) with a random systematic transrectal prostate biopsy $(\mathrm{SB})[3,4]$. Due to a high soft-tissue contrast, a high resolution (T2weighted anatomical sequences) and the registration of functional parameters (diffusion-weighted and dynamic contrast-enhanced sequences (DWI and DCE), MR spectroscopy imaging) a multiparametric magnetic resonance imaging (mpMRI) of the prostate provides a high sensitivity, specificity and negative predictive value in the detection and localization of clinically significant prostate cancers $[5,6]$. For standardization of evaluation of the mpMRI the "European Society of Urogenital Radiology" (ESUR) established the "Prostate Imaging Reporting and Data System" (PI-RADS), which introduced a 5-point Likert scale for each region (peripheral and central glandular sections) with corresponding scores for each sequence (T2, DWI, DCE, and MR-Spectroscopy) [7, 8]. The correlation of the level of PI-RADS with the overall detection rate of $\mathrm{PCa}$ and the detection of significant $\mathrm{PCa}$ has been demonstrated in various studies [9-13]. The increasing utilization of mpMRI of the prostate and the consecutive MRI/ultrasound fusion guided targeted biopsy (TB) resulted in an improved detection of PCa compared to SB, the current standard of care [14-17]. A difficulty is the exact fusion of mpMRI with transrectal ultrasound for TB. Various possibilities of MRI/ultrasound (MRI/US) image fusion, such as cognitive fusion, sensor-based fusion or organ-based fusion are available to perform TB. Despite the technological progress of different fusion platforms, several studies have shown that clinically significant $\mathrm{PCa}$ can still be overlooked by TB [17-20]. For the sensorbases $\mathrm{TB}$ we previously analyzed the possible pitfalls of $\mathrm{TB}$, such as reader variability for mpMRI, an imprecise targeting of the suspicious lesion [21]. Traditionally sensor-based fusion of the MRI image and the real-time ultrasound image is performed by the operator in the axial plane according to anatomical landmarks (i.e. prostatic apex, periprostatic vessels, BPH nodes etc.). In order to further improve the targeting accuracy and reduce a possible image fusion error, this study evaluated the use of an additional image fusion in the sagittal plane according to a 3-point technique. In a cohort of 791 men, who underwent a MRI/US fusion biopsy with an organ-based fusion system, Hong et al. demonstrated that the combination of axial and sagittal approaches detected more clinically significant $\mathrm{PCa}$ [22]. For sensor-based fusion platforms there is currently no data on the effect of an added sagittal image fusion.

\section{Methods}

\section{Study population}

In the period of July 2013 to September 2015, 251 patients, who showed at least one suspicious lesion on mpMRI (PI-RADS $\geq 3$ ) and underwent a consecutive TB in combination with a 10-core systematic prostate biopsy (SB), were consecutively included into the retrospective analysis. The indication for a mpMRI has largely been provided by attending outpatient urologists. Parts of the cohort were analysed in a previous study [13]. All patients were recorded regardless to the number of prior prostate biopsies. The data collection was based on the patients medical history, clinical findings and the physical patient files. Patient data was prospectively collected in a START conform database [23]. The analysis in regard to the axial and sagittal image fusion was performed retrospectively. All patients signed an informed consent for the intervention, data acquisition and data evaluation. The study was performed according to the declaration of Helsinki and the analysis was approved by the Institutional Review Board of the Charité University Medicine Berlin.

\section{Multiparametric magnetic resonance imaging}

A 3-Tesla mpMRI (Magnetom Skyra, Siemens Medical Systems, Erlangen, Germany) without an endorectal coil was performed for all patients before prostate biopsy. The MRI protocol contained high spatial resolution T2-weighted turbo spin-echo sequences in axial, sagittal and coronal orientation, axial turbo spin-echo $\mathrm{T} 1$ weighted images, axial diffusion weighted images (b-values 0.400 and $800 \mathrm{~s} / \mathrm{mm}^{2}$ ) and dynamic contrast-enhanced sequences. The evaluation of the mpMRI was performed by experienced radiologists according to the guidelines of the European Society of Urogenital Radiology (ESUR) [8]. From a PI-RADS score of 3 , the indication for MRI/US fusion biopsy was made.

\section{MRI-ultrasound fusion prostate biopsy and systematic biopsy}

The prostate biopsies were performed under antibiotic prophylaxis with a fluoroquinolone according to the EAU guidelines [2], with a high-end ultrasound device HiVision Preirus (Hitachi Medical Systems, Tokyo, Japan) and an endocavity endfire probe (EUP V53W, Hitachi Medical Systems, Tokyo, Japan). All biopsies were taken in lithotomy position. At first TB were performed. T2 and DWI sequences of the axial planes in mpMRI were imported to the ultrasonic device. After that, the suspicious lesions were marked in axial orientation of the mpMRI sequences by the urologist experienced with mpMRI. The MRI/US image fusion was performed using sensor-based registration. The movement of the probe with an attached tracker was detected in a low magnetic field (0.1 Tesla), which was generated by a mini bird receiver. Until December 2014 only axial MRI/US image fusion was performed. For 
this purpose, the same plane in ultrasound and MRI was identified according to anatomical landmarks (prostatic apex, periprostatic vessels, BPH nodes, intraprostatic cysts) Depending on the anatomical conditions, the angle of axial plane in the MRI image was corrected to match the angulation of the ultrasound probe and image. The previously marked suspect lesions were transferred to the real-time ultrasound image by the platform's software, followed by a $2-5$ targeted biopies in an axial orientation. After an analysis of possible reasons for targeted biopsy failure, as of December 2014 the targeted biopsies were performed after MRI/US image fusion in both the axial and sagittal plane [21]. The total number of targeted biopsies remained unchanged. For the sagittal image fusion, a T2-weighted sequence in sagittal orientation was used to mark the bladder neck, the apex of the prostate and the seminal vesicle angle. Based on these marks the MRI and the ultrasound image were fused by the software. Thereafter, TB was carried out in a sagittal orientation of the MRI and ultrasound image. For sampling, we used a long biopsy needle $(18 \mathrm{~g} \times 25 \mathrm{~cm}$, Bard Magnum biopsy instrument, Tempe, United States). After performing TB, local anaesthesia with a bupivacaine was injected at the dorsal prostatic capsule and a 10-core SB was conducted without changing the examiner. The SB scheme included cores from: left/right apex, left/right lateral mid gland, left/right base, left/right ventral and left/right para-urethral. All tissue-samples were documented by their extraction location and shipped separately for histopathological evaluation by our experienced pathologists.

\section{Group distribution}

Group A included all patients who have received an MRI/ US image fusion only in the axial plane. Group B, are included all patients who have received MRI/US image fusion in the axial and sagittal plane. Figure 1 shows a flow chart for the patient inclusion and the group distribution.

\section{Statistical analysis}

PASW Version 22 (SPSS Inc. 1998-2010, Chicago, Illinois 60606, USA) was used for statistical analyses. Categorical data were presented by absolute and relative frequencies.
Continuous variables were measured by means and standard deviation when normal distributed or by medians and quartiles. Continuous variables were evaluated with the Kolmogorov-Smirnov-test for normal distribution. We used chi-square test, Student's t-test and Mann-Whitney U test to calculate statistical differences between numerical and categorical variables. Univariate and multivariate binary regression analysis were performed to evaluate significant parameters in the descriptive analysis as predictors for PCa detection. A p-value of $p<0.05$ was considered statistical significant.

\section{Results}

Demographic data, clinical characteristics and MRI findings are presented in Table 1 divided in group A (patients without additional sagittal image fusion) and group B (patients with additional sagittal image fusion). The median age in group A was 67 years (IQR 61-72) and in group B 68 years (IQR 60-71). Both groups showed statistically similar prostate volumes (48 vs. $50 \mathrm{ml})$. The median PSA level in group A was $8.10 \mathrm{ng} /$ $\mathrm{ml}$ (IQR 6.05-14.00) and in group B $8.59 \mathrm{ng} / \mathrm{ml}$ (IQR 5.65-12.32). The proportion of patients with a suspicious digital rectal examination (DRE) (14 vs. $29 \%, p=$ 0.007 ) and the proportion of patients with primary biopsies ( 33 vs $46 \%, p=0.041$ ) were significantly lower in group A. The rate of PI-RADS 3 lesions were overrepresented in group A (19 vs. $9 \% ; p=0.044)$. With $43 \%$ compared to $30 \%$ in group A PI-RADS 5 lesions were more frequently represented in group B $(p=0.051)$. No significant differences were observed for lesion positions, number of suspicious lesions in mpMRI and lesion sizes. The mean number of cores taken per patient and the mean number of TB per patient were significant higher in Group A (13.7 vs. 13.2 and 3.8 vs. 3.4; $p=0.009$ and 0.031 ). The analysis showed a significant higher overall cancer detection rate (CDR) (85 vs. 72\%; $p=0.019)$ and a significant higher detection rate in TB (76 vs. $55 \% ; p=0.001$ ) in group $B$, please see Table 2 . Furthermore there was a significant lower number of patients diagnosed with a clinically significant $\mathrm{PCa}$ in group A (61 vs. $78 \% ; p=0.025$ ). Classified according to

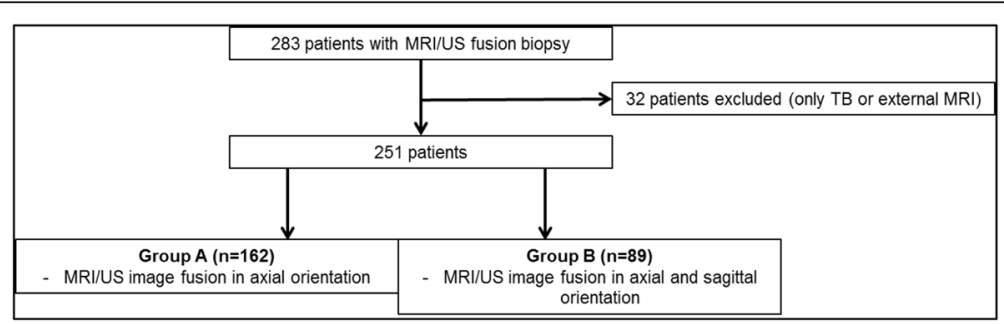

Fig. 1 Patient inclusion and group distribution. Group A included patients between July 2013 and December 2014 where an axial targeted biopsy was the standard Group B included patients between December 2014 and September 2015 where and axial and sagittal targeted biopsy was performed without increasing the total number of targeted biopsy cores 
Table 1 Patient demographics and magnetic resonance imaging findings $(n=251)$

\begin{tabular}{|c|c|c|c|}
\hline & $\begin{array}{l}\text { Group A } \\
(n=162)\end{array}$ & $\begin{array}{l}\text { Group B } \\
(N=89)\end{array}$ & $p$-value \\
\hline Median (IQR) age, years & $67(61-72)$ & $68(60-71)$ & 0.846 \\
\hline Median (IQR) PSA. ng/ml & $8.10(6.05-14.00)$ & $8.59(5.65-12.32)$ & 0.997 \\
\hline Median (QR) f/t PSA-ratio,\% & $12(9-17)$ & $13(9-19)$ & 0.309 \\
\hline $\begin{array}{l}\text { Median (IQR) prostate } \\
\text { volume, } \mathrm{ml}\end{array}$ & $48(35-60)$ & $50(37-70)$ & 0.087 \\
\hline Suspicous DRE, n (\%) & $23(14 \%)$ & $26(29 \%)$ & 0.007 \\
\hline \multicolumn{4}{|l|}{ No. of prior biopsies, n (\%) } \\
\hline Primary biopsy & $53(33 \%)$ & $41(46 \%)$ & 0.041 \\
\hline 1 & $59(36 \%)$ & $28(32 \%)$ & 0.489 \\
\hline 2 & $33(20 \%)$ & $13(15 \%)$ & 0.308 \\
\hline$\geq 3$ & $17(10 \%)$ & $7(8 \%)$ & 0.655 \\
\hline \multicolumn{4}{|l|}{$\begin{array}{l}\text { Localization of lesions with } \\
\text { maximum PI-RADS on mpMRI, } \\
n(\%)\end{array}$} \\
\hline Apex & $72(44 \%)$ & $35(39 \%)$ & 0.505 \\
\hline Midgland & $43(27 \%)$ & $32(36 \%)$ & 0.149 \\
\hline Base & $47(29 \%)$ & $22(25 \%)$ & 0.555 \\
\hline Anterior & $45(28 \%)$ & $28(32 \%)$ & 0.563 \\
\hline $\begin{array}{l}\text { Median (IQR) no. of lesions } \\
\text { per patient }\end{array}$ & $1(1-2)$ & $1(1-2)$ & 0.451 \\
\hline $\begin{array}{l}\text { Maximum diameter of } \\
\text { lesions }(\mathrm{mm})\end{array}$ & $14(10-17)$ & $13(10-18)$ & 0.885 \\
\hline $\begin{array}{l}\text { Mean (SD) of cores taken } \\
\text { per patient }\end{array}$ & $13.7( \pm 1.6)$ & $13.2( \pm 1.2)$ & 0.009 \\
\hline Mean (SD) TBs per patient & $3.8( \pm 1.5)$ & $3.4( \pm 0.9)$ & 0.031 \\
\hline \multicolumn{4}{|l|}{$\begin{array}{l}\text { Maximum mpMRI Score, } \\
\mathrm{n}(\%)^{\mathrm{a}}\end{array}$} \\
\hline PI-RADS 3 & $31(19 \%)$ & $8(9 \%)$ & 0.044 \\
\hline PI-RADS 4 & 83 (51\%) & $43(48 \%)$ & 0.693 \\
\hline PI-RADS 5 & 48 (30\%) & 38 (43\%) & 0.051 \\
\hline
\end{tabular}

$P S A$ prostate-specific antigen, IQR interquartile range, $S D$ standard deviation, $D R E$ digital rectal examination, $m p M R I$ multiparametric magnetic resonance imaging, PI-RADS Prostate Imaging Reporting and Data System, TB targeted biopsy

${ }^{\text {a}}$ For patients with multiple lesions the highest PI-RADS score is stated

PI-RADS 3, 4 and 5, the detection rates of TB were 42 , $48,75 \%$ in group $\mathrm{A}$ and $25,74,90 \%$ in group $\mathrm{B}$. The rate of PCa with a Gleason score $\geq 7$ missed by TB was $33 \%$ (18 cases) in group A and $\mathbf{9 \%}$ (5 cases) in group B ( $p=$ 0.072). The overall cancer detection rates and the PIRADS based analyses for SB and TB for men without suspicious DRE and prior negative biopsy are shown in Additional file 1 Table S1 and Additional file 2 Table S2. An explorative multivariate binary logistic regression analysis revealed that PI-RADS, a suspicious DRE and performing an additional sagittal image fusion were significant predictors for $\mathrm{PCa}$ detection in $\mathrm{TB}$, please see
Table 2 Cancer Detection Rate and Gleason pattern

\begin{tabular}{|c|c|c|c|}
\hline & $\begin{array}{l}\text { Group A } \\
(n=162)\end{array}$ & $\begin{array}{l}\text { Group B } \\
(N=89)\end{array}$ & $p$-value \\
\hline Overall CDR & $117(72 \%)$ & $76(85 \%)$ & 0.019 \\
\hline SB & $108(67 \%)$ & $68(76 \%)$ & 0.115 \\
\hline $\mathrm{TB}$ & $89(55 \%)$ & $68(76 \%)$ & 0.001 \\
\hline \multicolumn{4}{|l|}{ PI-RADS $3(n=39)$} \\
\hline Overall CDR & $17(55 \%)$ & $5(63 \%)$ & $>0.999$ \\
\hline SB & 15 (48\%) & $3(38 \%)$ & 0.702 \\
\hline TB & $13(42 \%)$ & $2(25 \%)$ & 0.450 \\
\hline \multicolumn{4}{|l|}{ PI-RADS $4(n=126)$} \\
\hline Overall CDR & $55(66 \%)$ & $36(84 \%)$ & 0.058 \\
\hline SB & $48(58 \%)$ & $33(77 \%)$ & 0.049 \\
\hline TB & 40 (48\%) & $32(74 \%)$ & 0.007 \\
\hline \multicolumn{4}{|l|}{ PI-RADS $5(n=86)$} \\
\hline Overall CDR & 45 (94\%) & $35(92 \%)$ & $>0.999$ \\
\hline SB & 45 (94\%) & $32(84 \%)$ & 0.175 \\
\hline TB & $36(75 \%)$ & 34 (90\%) & 0.102 \\
\hline Detected GS $\geq 7$ in TB & $54(61 \%)$ & $53(78 \%)$ & 0.025 \\
\hline Missed PCa $(G S \geq 7)$ in $T B$ & $18(33 \% *)$ & $5(9 \% *)$ & 0.072 \\
\hline
\end{tabular}

$\mathrm{CDR}=$ Cancer Dection Rate; GS = Gleason Score; SB = Random Biopsy; TB = Target biopsy; * \% of GS $\geq 7$ detected by TB

Table 3. Table 4 shows the comparison of the biopsy results of the axial (aTB) and sagittal (sTB) MRI/US fusion biopsy. Depending on PI-RADS, lesion diameter and lesion localization, $\mathrm{PCa}$ detection rates of aTB and sTB were statistically equivalent except a higher detection of PCa by aTB for PI-RADS 4 lesions (70 vs. 44\%, $p=$ 0.007). Furthermore, Table 4 shows the additional detection of PCa in total and of PCa with a Gleason score $\geq 7$ due sTB depending on PI-RADS, lesion diameter and lesion localization. Overall, nine PCas were only detected by sTB and sTB identified 10 additional clinically significant PCa (Gleason $\geq 7$ ).

\section{Discussion}

Since the introduction of MRI/US fusion biopsy of the prostate, several studies have demonstrated an improvement in prostate cancer detection rates as well as the identification of clinically relevant tumours [14, 15, 24-26]. Due to this increasing value of MRI/US fusion biopsy for

Table 3 Predictors for prostate cancer detection in the Targeted Biopsy

\begin{tabular}{llllll}
\hline & \multicolumn{2}{l}{ Univariate analysis } & & \multicolumn{2}{c}{ Multivariate analysis } \\
\cline { 2 - 3 } & OR & $p$-value & & OR & $p$-value \\
\hline Suspicious DRE & 4.539 & $<0.001$ & & 2.777 & 0.024 \\
Primary biopsy & 1.175 & 0.553 & & \\
PI-RADS & 2.712 & $<0.001$ & & 2.240 & $<0.001$ \\
Sagittal image fusion & 2.656 & 0.001 & & 2.105 & 0.017 \\
\hline
\end{tabular}


Table 4 Cancer Detection Rate of the Targeted Biopsy in relation to an axial and sagittal image fusion

\begin{tabular}{|c|c|c|c|c|c|}
\hline Group B $n=89$ & Overall CDR in TB (sTB + aTB) & CDR in aTB & CDR in STB & $\begin{array}{l}\text { Additional PCa detected } \\
\text { only by sTB* }\end{array}$ & $\begin{array}{l}\text { Additional GS } \geq 7 \\
\text { in } S^{\# B^{\#}}\end{array}$ \\
\hline Overall & $68(76 \%)$ & $59(66 \%)$ & $50(56 \%)$ & $9(13 \%)$ & $10(19 \%)$ \\
\hline \multicolumn{6}{|l|}{ PI-RADS } \\
\hline $3(n=8)$ & $2(25 \%)$ & $2(25 \%)$ & $2(25 \%)$ & 0 & 0 \\
\hline $4(n=43)$ & $32(74 \%)$ & $30(70 \%)$ & $19(44 \%)$ & $2(5 \%)$ & $4(9 \%)$ \\
\hline $5(n=38)$ & $34(90 \%)$ & $27(71 \%)$ & $29(76 \%)$ & $7(18 \%)$ & $6(16 \%)$ \\
\hline \multicolumn{6}{|c|}{ Maximum diameter of lesion } \\
\hline $1-10(n=27)$ & $18(67 \%)$ & 15 (56\%) & $10(37 \%)$ & $3(11 \%)$ & $3(11 \%)$ \\
\hline $11-20(n=50)$ & $38(76 \%)$ & $33(66 \%)$ & $29(58 \%)$ & $5(10 \%)$ & $5(10 \%)$ \\
\hline$>20(n=12)$ & $12(100 \%)$ & $11(92 \%)$ & $11(92 \%)$ & $1(8 \%)$ & $2(17 \%)$ \\
\hline \multicolumn{6}{|l|}{ Localization of lesion } \\
\hline Apex $(n=35)$ & $26(74 \%)$ & $23(66 \%)$ & 19 (54\%) & $3(9 \%)$ & $3(9 \%)$ \\
\hline Midgland $(n=32)$ & $23(72 \%)$ & $18(56 \%)$ & $15(47 \%)$ & $5(16 \%)$ & $5(16 \%)$ \\
\hline Base $(n=22)$ & 19 (86\%) & 18 (82\%) & $16(73 \%)$ & $1(5 \%)$ & $2(9 \%)$ \\
\hline Anterior $(n=28)$ & $26(93 \%)$ & 22 (79\%) & $22(79 \%)$ & $4(14 \%)$ & $5(18 \%)$ \\
\hline
\end{tabular}

aTB = axial fusion TB; sTB = sagittal fusion TB; * compared to overall CDR or TB (aTB + sTB);

\#Either detection of GS $\geq 7$ only by sTB or Gleason upgrade in the sTB biopsy core compared to the aTB core; percentage of GS $\geq 7$ detected by TB (aTB + sTB) $n=53$

primary diagnosis and monitoring of prostate cancer various fusion systems have been established. A variety fusion systems (UroNav, BiopSee, Urostation, Artemis, HiVisonPreirus, etc.) have been reported in the current literature $[13,14,22,24,26,27]$. Uniform treatment regimens for the implementation of MRI/US fusion biopsies do not exist. In a large patient cohort Hong et al. demonstrated for organ based MRI/US fusion biopsies that the combination of sagittal and axial biopsy approaches identified additional clinically significant prostate cancers [22]. It can be assumed that the correctness of the image fusion of MRI and transrectal ultrasound has an important influence on the accuracy of targeted sampling. Our study showed a significant increase in prostate cancer detection rate of TB by $55 \%$ in the group without sagittal fusion to $76 \%$ in the group with additional sagittal fusion and the improvement remained even when men with a positive DRE and a primary biopsy were excluded. In addition, the proportion of detected clinically significant $\mathrm{PCa}$ (Gleason-score $\geq 7$ ) increased from $61 \%$ in group A to $78 \%$ in group B. The sole analysis of the detection rates of axial TB results in an increase of 56 to $66 \%$ in group B. The observed increase in detection rates might be related to various factors. In the sensor-based MRI/US image fusion, the same layers in the T2-weighted MRI sequence and the transrectal ultrasound image in axial or sagittal orientation are fused. Identifying the same layers in MRI and US are the basis of fusion accuracy. Angular deviations of the display plane in transrectal ultrasound and MRI lead to inaccuracies of image fusion. In our study, the angle correction for axial image fusion was carried out manually by the urologist. In the sagittal image fusion, the angular offset is software-based by three identical points, which are simultaneously marked in MRI and ultrasound in two different layers. Gaziev et al. showed an increase in the detection rate of prostate cancer by performing perineal MRI/US fusion biopsies of the prostate with increasing experience of the examiner [28]. It is tempting to speculate, that in our study the learning curve of the examiner has likewise lead to an increase in the detection rate in the TB after implementation of additional sagittal image fusion. Another important factor influencing the detection rate of TB is the PI-RADS score $[12,13]$. Our study cohort showed a significant decrease of percentage of PI-RADS 3 lesions and a non-significant increase in the proportion of PIRADS 5 lesions in the patient group with additional sagittal fusion. Also in the univariate and multivariate regression analysis the level of PI-RADS was identified as a significant predictor for $\mathrm{PCa}$ detection. This may have occurred to an increased PCa detection in group B, but the sagittal image fusion remained an independent predictor for cancer detection by TB. A suspicious digital rectal examination as described by Radtke et al. and Potter et al. presents a further risk factor for the detection of PCa in TB and SB $[18,29]$. Similar to the previously published studies, our univariate and multivariate regression analyses of the whole cohort revealed a significant correlation of a suspicious DRE with $\mathrm{PCa}$ detection rate. The higher TB detection rate in group $\mathrm{B}$, that included more men with a suspicious DRE, may therefore have been influenced, but the higher detection rate in group B compared to group A persisted when the analysis excluded men with a suspicious DRE. In our regression analysis, the proportion of biopsy naive men was not a significant predictor for $\mathrm{PCa}$ detection, although two large studies showed an influence on cancer detection 
$[12,22]$. Therefore, the significantly higher proportion of primary biopsies in group B may have affected the detection rate of the TB. Again, the improved detection rate in group $B$ remained when men with a positive DRE and a primary biopsy were excluded from the analysis.

The additional implementation of the sagittal image fusion resulted in an increase in the detection rate of $10 \%$ for TB. By sagittal fusion, nine (13\%) additional prostate cancers were detected and ten (19\%) additional clinically significant tumors were identified. The improvement of the axial TB, when adding a sagittal TB was independent of the lesion size or localization of the lesions. Moreover, performing a sagittal image fusion was a significant predictor in univariate and multivariate regression analysis for the detection of prostate cancer in the TB. In the group of patients with sagittal fusion, the proportion of overlooked clinically significant tumors by TB dropped to $9 \%$ compared to $33 \%$ in the group of patients without sagittal fusion. The reduced rate of missed PCa after the introduction of the sagittal image fusion was not accompanied with an increase of the number of targeted biopsies.

Adding the sagittal image fusion when performing TB on a sensor-based platform may reduce the targeting error that may be inevitable in some cases [21]. In our institution we have therefore established the additional sagittal image fusion firmly in our MRI/US fusion biopsy protocol.

Because of the retrospective study design the investigation has several limitations. Unconsidered confounders may have also influenced the study results, e.g. a selection bias of patients by referring outpatient urologist. The inhomogeneity of the two study groups in terms of baseline characteristics may have affected the study results. To ensure the data consistency, we performed logistic regression analyses for the evaluation of predictors of PCa detection by targeted biopsy. In order to clearly demonstrate the impact of an additional sagittal image fusion on the detection rate of TB would require prospective randomized studies.

\section{Conclusion}

Performing a sagittal image fusion in addition to the standard the axial fusion improves the accuracy to detect PCa by targeted biopsies performed with a sensor-based MRI/US fusion platform.

\section{Additional files}

Additional file 1: Table S1. Cancer Detection Rates in Group A and B excluding men with abnormal DRE. (DOCX 19 kb)

Additional file 2: Table S2. Cancer Detection Rate and Gleason pattern in Group A and B excluding men with abnormal DRE and including only men with prior negative biopsy. (DOCX $19 \mathrm{~kb}$ )

\section{Abbreviations}

DCE: Dynamic contrast-enhanced sequences; DWI: Diffusion-weighted; ESUR: European society of urogenital radiology; mpMRI: Multiparametric magnetic resonance imaging; MRI/US: MRI/ultrasound; PCa: Prostate cancer SB: Systematic transrectal prostate biopsy; TB: MRI/ultrasound fusion guided targeted biopsy

\section{Acknowledgements}

None.

Funding

None.

\section{Availability of data and materials}

The datasets during and/or analysed during the current study available from the corresponding author on reasonable request.

\section{Authors' contributions}

KG, HC, CK: Protocol/project development. KG, HC, JB, MK, PA, MH: Data collection or management. KG, HC, CK: Data analysis. KG, HC, CK: Manuscript writing/editing. PA, JN, SH, KM: Critical manuscript revision. JN, SH, KM, CK: Supervision.

\section{Competing interests}

$\mathrm{H}$. Cash reports receiving honoraria as a speaker on national conferences for Hitachi Medical Systems. All other authors have no competing interests.

\section{Consent for publication}

Not applicable.

\section{Ethics approval and consent to participate}

All patients signed an informed consent for the intervention, data acquisition and data evaluation. The study was performed according to the declaration of Helsinki. The analysis was approved by the Institutional Review Board of the Charité University Medicine Berlin.

\section{Author details}

'Department of Urology, Charité - University Medicine Berlin, Hindenburgdamm 30, 12203 Berlin, Germany. ²Departement of Radiology, Charité — University Medicine Berlin, Hindenburgdamm 30, 12203 Berlin, Germany.

Received: 8 July 2016 Accepted: 23 December 2016

Published online: 13 January 2017

\section{References}

1. Siegel RL, Miller KD, Jemal A. Cancer statistics, 2015. CA Cancer J Clin. 2015; 65(1):5-29.

2. Heidenreich A, Bastian PJ, Bellmunt J, Bolla M, Joniau S, van der Kwast T, et al. EAU guidelines on prostate cancer. part 1: screening, diagnosis, and local treatment with curative intent-update 2013. Eur Urol. 2014:65(1):124-37.

3. Rodriguez-Covarrubias F, Gonzalez-Ramirez A, Aguilar-Davidov B, CastillejosMolina R, Sotomayor M, Feria-Bernal G. Extended sampling at first biopsy improves cancer detection rate: results of a prospective, randomized trial comparing 12 versus 18-core prostate biopsy. J Urol. 2011;185(6):2132-6.

4. Campos-Fernandes JL, Bastien L, Nicolaiew N, Robert G, Terry S, Vacherot F, et al. Prostate cancer detection rate in patients with repeated extended 21-sample needle biopsy. Eur Urol. 2009;55(3):600-6.

5. Futterer JJ, Briganti A, De Visschere P, Emberton M, Giannarini G, Kirkham A, et al. Can clinically significant prostate cancer Be detected with multiparametric magnetic resonance imaging? a systematic review of the literature. Eur Urol. 2015:68(6):1045-53.

6. Arumainayagam N, Ahmed HU, Moore CM, Freeman A, Allen C, Sohaib SA, et al. Multiparametric MR imaging for detection of clinically significant prostate cancer: a validation cohort study with transperineal template prostate mapping as the reference standard. Radiology. 2013;268(3):761-9.

7. Hamoen EH, de Rooij M, Witjes JA, Barentsz JO, Rovers MM. Use of the prostate imaging reporting and data system (PI-RADS) for prostate cancer detection with multiparametric magnetic resonance imaging: a diagnostic meta-analysis. Eur Urol. 2015;67(6):1112-21.

8. Barentsz JO, Richenberg J, Clements R, Choyke P, Verma S, Villeirs G, et al. ESUR prostate MR guidelines 2012. Eur Radiol. 2012;22(4):746-57.

9. Arsov C, Rabenalt R, Blondin D, Quentin M, Hiester A, Godehardt E, et al. Prospective randomized trial comparing magnetic resonance imaging 
(MRI)-guided in-bore biopsy to MRI-ultrasound fusion and transrectal ultrasound-guided prostate biopsy in patients with prior negative biopsies. Eur Urol. 2015;68(4):713-20

10. Baco E, Rud E, Eri LM, Moen G, Vlatkovic L, Svindland A, et al. A Randomized Controlled Trial To Assess and Compare the Outcomes of Two-core Prostate Biopsy Guided by Fused Magnetic Resonance and Transrectal Ultrasound Images and Traditional 12-core Systematic Biopsy. Eur Urol. 2016;69(1):149-56.

11. Borkowetz A, Platzek I, Toma M, Laniado M, Baretton G, Froehner M, et al. Comparison of systematic transrectal biopsy to transperineal magnetic resonance imaging/ultrasound-fusion biopsy for the diagnosis of prostate cancer. BJU Int. 2015;116(6):873-9.

12. Filson CP, Natarajan S, Margolis DJ, Huang J, Lieu P, Dorey FJ, et al. Prostate cancer detection with magnetic resonance-ultrasound fusion biopsy: The role of systematic and targeted biopsies. Cancer. 2016;122(6):884-92.

13. Cash H, Maxeiner A, Stephan C, Fischer T, Durmus T, Holzmann J, et al. The detection of significant prostate cancer is correlated with the Prostate Imaging Reporting and Data System (PI-RADS) in MRI/transrectal ultrasound fusion biopsy. World J Urol. 2016;34(4):525-32.

14. Siddiqui MM, Rais-Bahrami S, Turkbey B, George AK, Rothwax J, Shakir N, et al. Comparison of MR/ultrasound fusion-guided biopsy with ultrasound-guided biopsy for the diagnosis of prostate cancer. JAMA. 2015;313(4):390-7.

15. Siddiqui MM, Rais-Bahrami S, Truong H, Stamatakis L, Vourganti S, Nix J, et al. Magnetic resonance imaging/ultrasound-fusion biopsy significantly upgrades prostate cancer versus systematic 12-core transrectal ultrasound biopsy. Eur Urol. 2013;64(5):713-9.

16. Pokorny MR, de Rooij M, Duncan E, Schroder FH, Parkinson R, Barentsz JO, et al. Prospective study of diagnostic accuracy comparing prostate cancer detection by transrectal ultrasound-guided biopsy versus magnetic resonance (MR) imaging with subsequent MR-guided biopsy in men without previous prostate biopsies. Eur Urol. 2014;66(1):22-9.

17. Salami SS, Ben-Levi E, Yaskiv O, Ryniker L, Turkbey B, Kavoussi LR, et al. In patients with a previous negative prostate biopsy and a suspicious lesion on magnetic resonance imaging, is a 12-core biopsy still necessary in addition to a targeted biopsy? BJU Int. 2015;115(4):562-70.

18. Radtke JP, Kuru TH, Boxler S, Alt CD, Popeneciu IV, Huettenbrink C, et al. Comparative analysis of transperineal template saturation prostate biopsy versus magnetic resonance imaging targeted biopsy with magnetic resonance imaging-ultrasound fusion guidance. J Urol. 2015;193(1):87-94.

19. Distler F, Radtke JP, Kesch C, Roethke M, Schlemmer HP, Roth W, et al. [Value of MRI/ultrasound fusion in primary biopsy for the diagnosis of prostate cancer]. Urologe A. 2016;55(2):146-55.

20. Radtke JP, Schwab C, Wolf MB, Freitag MT, Alt CD, Kesch C, et al. Multiparametric Magnetic Resonance Imaging (MRI) and MRI-Transrectal Ultrasound Fusion Biopsy for Index Tumor Detection: Correlation with Radical Prostatectomy Specimen. Eur Urol. 2016;70(5):846-853.

21. Cash H, Gunzel K, Maxeiner A, Stephan C, Fischer T, Durmus T, et al. Prostate cancer detection on transrectal ultrasonography-guided random biopsy despite negative real-time magnetic resonance imaging/ultrasonography fusion-guided targeted biopsy: reasons for targeted biopsy failure. BJU Int. 2016;118(1):35-43.

22. Hong CW, Rais-Bahrami S, Walton-Diaz A, Shakir N, Su D, George AK, et al. Comparison of magnetic resonance imaging and ultrasound (MRI-US) fusionguided prostate biopsies obtained from axial and sagittal approaches. BJU Int. 2015;115(5):772-9.

23. Moore CM, Kasivisvanathan V, Eggener S, Emberton M, Futterer JJ, Gill IS, et al. Standards of reporting for MRI-targeted biopsy studies (START) of the prostate: recommendations from an international working group. Eur Urol. 2013;64(4):544-52.

24. Sonn GA, Chang E, Natarajan S, Margolis DJ, Macairan M, Lieu P, et al. Value of targeted prostate biopsy using magnetic resonance-ultrasound fusion in men with prior negative biopsy and elevated prostate-specific antigen. Eur Urol. 2014;65(4):809-15.

25. Wysock JS, Rosenkrantz AB, Huang WC, Stifelman MD, Lepor H, Deng FM, et al. A prospective, blinded comparison of magnetic resonance (MR) imaging-ultrasound fusion and visual estimation in the performance of MR-targeted prostate biopsy: the PROFUS trial. Eur Urol. 2014;66(2):343-51.

26. Delongchamps NB, Peyromaure M, Schull A, Beuvon F, Bouazza N, Flam T, et al. Prebiopsy magnetic resonance imaging and prostate cancer detection: comparison of random and targeted biopsies. J Urol. 2013;189(2):493-9.
27. Kuru TH, Roethke MC, Seidenader J, Simpfendorfer T, Boxler S, Alammar K, et al. Critical evaluation of magnetic resonance imaging targeted, transrectal ultrasound guided transperineal fusion biopsy for detection of prostate cancer J Urol. 2013;190(4):1380-6.

28. Gaziev G, Wadhwa K, Barrett T, Koo BC, Gallagher FA, Serrao E, et al. Defining the learning curve for multiparametric magnetic resonance imaging (MRI) of the prostate using MRI-transrectal ultrasonography (TRUS) fusion-guided transperineal prostate biopsies as a validation tool. BJU Int. 2016;117(1):80-6.

29. Potter SR, Horniger W, Tinzl M, Bartsch G, Partin AW. Age, prostate-specific antigen, and digital rectal examination as determinants of the probability of having prostate cancer. Urology. 2001;57(6):1100-4.

\section{Submit your next manuscript to BioMed Central and we will help you at every step:}

- We accept pre-submission inquiries

- Our selector tool helps you to find the most relevant journal

- We provide round the clock customer support

- Convenient online submission

- Thorough peer review

- Inclusion in PubMed and all major indexing services

- Maximum visibility for your research

Submit your manuscript at www.biomedcentral.com/submit
) Biomed Central 